\title{
Deconvolving the Galactic Centre with IBIS
}

\author{
V.A. McBride*, A.J. Bird and D.J. Clark \\ University of Southampton \\ E-mail: vanessa@soton.ac.uk,A.J.Bird@soton.ac.uk,djc4 $00 @$ soton.ac.uk
}

\section{A.B. Hill}

Laboratoire d'Astrophysique de Grenoble

Email: adam.hill@obs.ujf-grenoble.fr

\section{on behalf of the IBIS survey team}

\begin{abstract}
With a square degree field containing thousands of soft X-ray sources, the Galactic Centre source population presents a unique challenge to the 12 arcminute angular resolution of the IBIS telescope. Motivated by the notable variability of Galactic sources as observed in soft X-rays, we have employed combined temporal and spatial analyses of this region to generate a consistent set of sources describing the $20-40 \mathrm{keV}$ flux from the Galactic Centre over the lifetime of the INTEGRAL satellite. From this work follows a description of both the timing and spectral variability of these individual sources in an energy range poorly studied up until now, bringing us a step closer to identifying the types of sources emitting hard X-rays in our Galactic Centre.
\end{abstract}

The Extreme sky: Sampling the Universe above $10 \mathrm{keV}$ - extremesky2009, October 13-17, 2009

Otranto (Lecce) Italy

\footnotetext{
${ }^{*}$ Speaker.
} 

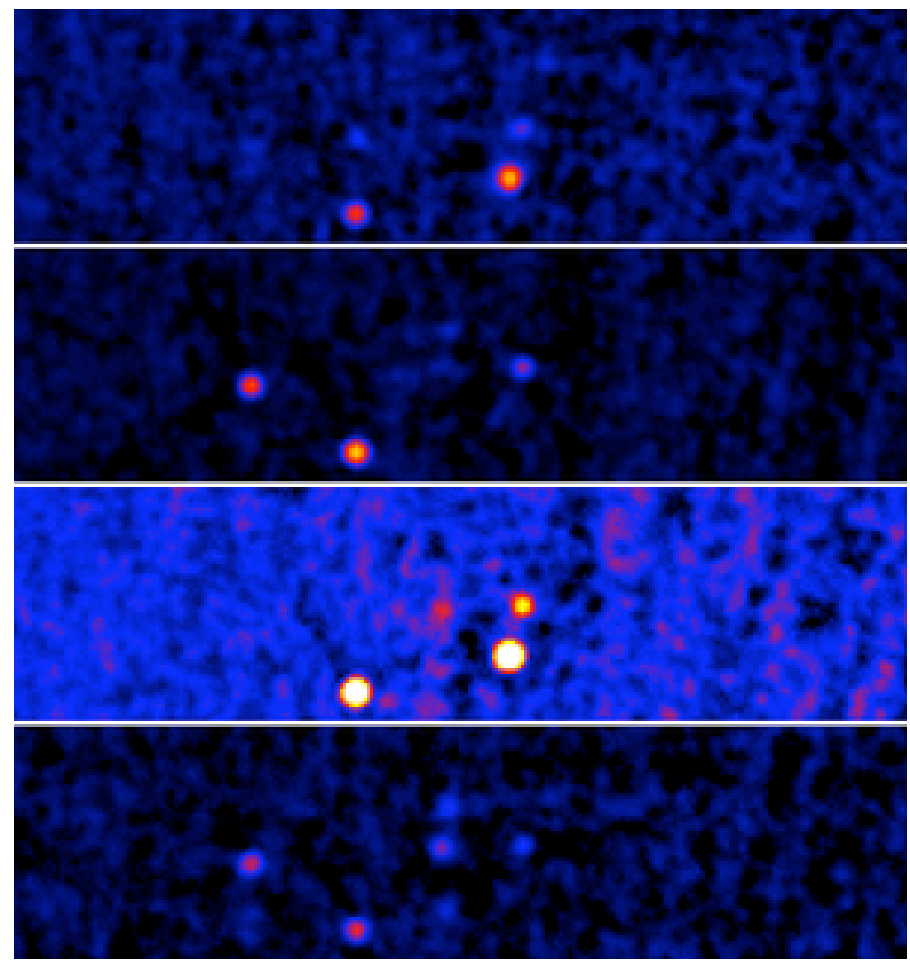

Figure 1: JEM-X observations of four epochs (from top to bottom: Feb-Apr 2004, Feb-Apr 2005, Feb-Apr 2006 and Feb-Apr 2007) of deep exposures of the Galactic Centre shown to highlight the source variability of the population. Observations are centred at $l=0, b=0$ and are $3.6 \times 0.8$ degrees in size with North up and $l$ increasing to the right.

\section{Introduction}

The Galactic Centre, with its crowded source population, poses a unique challenge to the 12 arcminute angular resolution of the IBIS telescope [6] aboard INTEGRAL. Muno et al., [5] have identified over $2000 \mathrm{X}$-ray point sources within a $17 \times 17 \mathrm{arcmin}^{2}$ field around Sgr A*, i.e. almost within an IBIS point-spread function. Although deep observations of this region have been presented $[2,1]$, and have highlighted the detection of $\mathrm{Sgr} \mathrm{A}^{*}$ in the $20-100 \mathrm{keV}$ waveband, the work presented here centres on understanding the source population of the Galactic Centre through utilising the variability of many of the sources that make up this population. The situation is well illustrated in Fig. 1, which shows deep exposure mosaics of the Galactic Centre with JEM-X at various epochs. The variability of the population is clearly visible. This variability, together with the spatial information given by IBIS in the $20-40 \mathrm{keV}$, is used to construct a list of point sources contributing to the observed flux in this energy range.

\section{Methods}

The IBIS mosaics of each satellite revolution in which the Galactic Centre was observed were used in the construction of the 4th IBIS Catalogue [3]. The mosaics were generated in the 20$40 \mathrm{keV}$ energy band and further details of the data processing and analysis can be found in [3]. The 

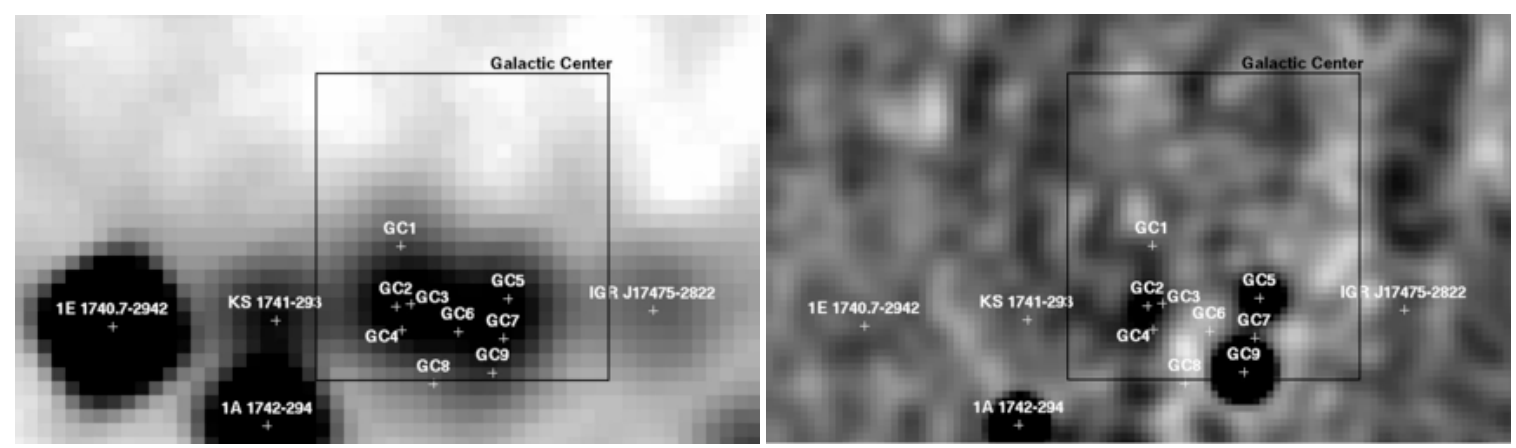

Figure 2: The fit region (rectangle) and the source population derived from the fits to each revolution. The IBIS 20-40 keV image appears in the left panel, while the JEM-X 3-10 keV deep exposure from FebApr 2006 appears in the right panel, showing clearly the spatial separation between 1E 1743.1-2843, SAX J1747.0-2853 and IGR J17456-2901. The images are oriented with North up and $l$ increasing to the right.

fit region is a $50.4 \times 52.8$ arcminute $^{2}$ rectangle centred at $l=0.12^{\circ}, b=0.18^{\circ}$, as shown by the rectangle in Fig. 2.

A core set of three sources, 1E 1743.1-2843, SAX J1747.0-2853 and IGR J17456-2901 were used as a starting point, the evidence of their presence from simultaneous, spatially wellseparated detection with JEM-X during a deep Galactic Centre exposure performed in satellite revolutions 407 through 429 (Feb - April 2006). For each revolution these three sources were fit as two dimensional Gaussian functions with their positions fixed to those in [4], FWHM fixed to 5.0 pixels (the IBIS point spread function of the mosaics in sky pixels) and normalisations free to vary. If $\chi_{v}^{2}>>1$, and a significant $(>3 \sigma)$ excess was present in the residuals, a new Gaussian was added with both position and normalisation free to vary. In addition to estimating the background from the fit, we used three regions of the same size as the fit region around the Galactic centre, centred at $(l, b)=\left(0.16^{\circ},-0.840^{\circ}\right),\left(358.960^{\circ}, 0.760^{\circ}\right)$ and $\left(356.959^{\circ},-1.040^{\circ}\right)$ for checking both significance of any local excesses, and the $\chi_{w}^{2}$ of the fits. This is due to the fact that during some revolutions the fit appears to be good, but the reduced chi-squared is $>>1-$ most likely as a result of systematic structures in the background. All three background regions were first checked for the presence of sources and then fit with a constant flux, in this way a 'target' $\chi_{a}^{2}$ was estimated from the average $\chi_{v}^{2}$ in these background regions.

The results for all revolutions are presented in Table 1 and Fig. 2. Those sources marked with a $\mathbf{Y}$ are new sources identified in this work.

\section{Characterising Variability}

A further, independent attempt at understanding how the Galactic Centre variability aids our understanding of the source population in this region was made by looking at a grid of $23 \times 26$ pixels covering the region shown in Fig. 2. For each pixel, a light curve at science window time resolution was generated, and three diagnostics (weighted mean, $\chi_{a}^{2}$ and bursticity ${ }^{1}$ ) were used to

\footnotetext{
${ }^{1}$ Bursticity is defined as the ratio of maximum significance on any timescale to the significance of the whole lightcurve. See [3] for further description.
} 


\begin{tabular}{lll}
\hline Identifier & Name & New \\
\hline GC1 & GRS 1741.9-2853 & $\mathrm{N}$ \\
GC2 & IGR J17456-2901 & $\mathrm{N}$ \\
GC3 & IGR J17457-2858 & $\mathrm{Y}$ \\
GC4 & IGR J17459-2902 & $\mathrm{Y}$ \\
GC5 & 1E 1743.1-2843 & $\mathrm{N}$ \\
GC6 & IGR J17463-2854 & $\mathrm{Y}$ \\
GC7 & IGR J17467-2848 & $\mathrm{Y}$ \\
GC8 & IGR J17468-2902 & $\mathrm{Y}$ \\
GC9 & SAX J1747.0-2853 & $\mathrm{N}$ \\
\hline
\end{tabular}

Table 1: Sources derived through the fitting procedure

explore the time variability of each pixel.

The weighted mean significance of the grid of pixels is dominated by IGR J17456-2901 and 1E 1743.1-2843 and gives an indication of the sources whose behaviours are closest to persistent throughout the time period studied. The $\chi_{v}^{2}$ of the pixel lightcurves is greatest around the positions of sources GRS 1741.9-2853 and SAX J1747.0-2853. These two sources have shown large outbursts during the monitoring period and hence the $\chi_{a}^{2}$ diagnostic gives a good indication of variable sources. The bursticity map, which is provided as a map of burst timescales, also highlights GRS 1741.9-2853 and SAX J1747.0-2853 as those with short burst timescales ( $\sim 10$ s of days), with the rest of the pixel lightcurves in the fit region show a typical bursticity timescale of $\sim 2000$ days, consistent with persistent emission from these sources.

None of the other transient sources detected in the fits to individual revolutions is present in the plots of Fig. 3. Whereas the fitting procedure described in the previous section accounts for the flux from neighbouring sources in each pixels, the pixel science window lightcurves used in this grid do not, and as such, the bright persistent sources of IGR J17456-2901 and 1E 1743.1-2843 dominate the surrounding pixels where new, faint, transient sources have been discovered. The fact that these new sources are typically much fainter than the two most obvious transients further impairs our ability to detect with this variability analysis.

\section{Results for individual sources}

Once the fitting procedure had been automated to account for all possible sources in Table 1, the behaviour of individual sources could be studied across the entire dataset. In Fig. 4 we present outbursts from the transient sources GRS 1741.9-2853 and SAX J1747.0-2853 observed during these observations. These lightcurves are made at revolution timescales in the $20-40 \mathrm{keV}$ energy. Together with lightcurves in the $40-100 \mathrm{keV}$ energy range, any changes in the spectra can be observed through outbursts, and more generally, as a function of time, for persistent sources in the fit region. In general the outbursts shown here tend to be harder when they become brighter. Future work will show whether such spectral changes observed in the $20-100 \mathrm{keV}$ band can be used to recognise differences between various types of sources. 

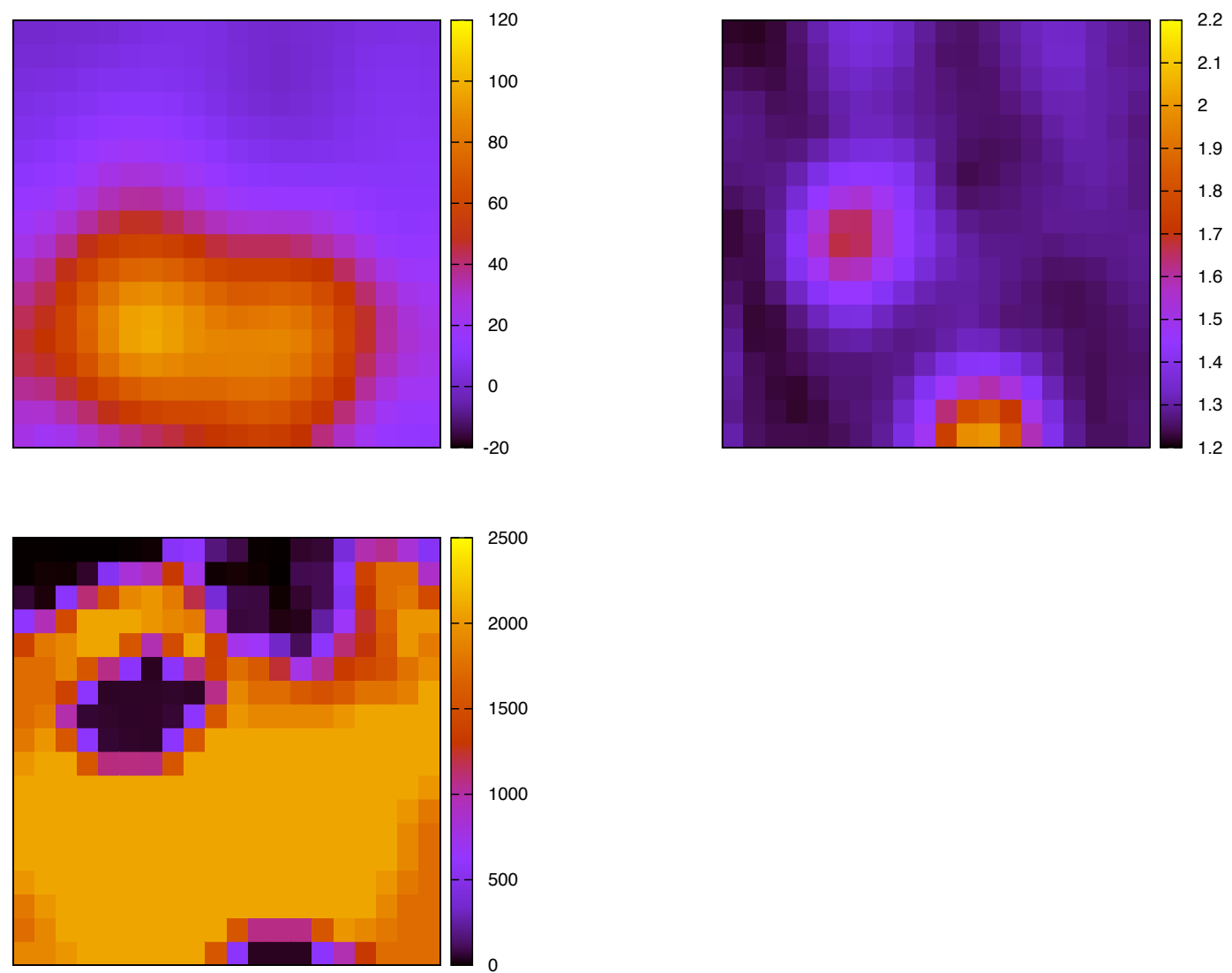

Figure 3: These plots show the weighted mean significance (top left), the $\chi_{v}^{2}$ fit to a straight line (top right) and the bursticity timescales (bottom left, in days) of the pixels in the Galactic Centre fit region at science window time resolution.

\section{Conclusion and future work}

In this work we have used both spatial and temporal information from 7.7 Ms of IBIS observations in the $20-40 \mathrm{keV}$ energy band to construct a coherent set of sources that explain the flux and variability of this flux in the central $1^{\circ} \times 1^{\circ}$ of our Galaxy.

These results represent a work in progress and there are a number of ways in which this analysis can be improved and extended. Some of these include refining of the IBIS point spread function instead of approximation by a Gaussian function, identifying the new hard X-ray sources in the Galactic Centre through their variability behaviour and through contemporaneous observations at soft X-ray wavelengths. It may be useful also to have lightcurves for these sources on shorter timescales, such as science windows, which will allow source variability on different timescales to be probed.

\section{References}

[1] Bélanger G., Goldwurm A., Renaud M., Terrier R., Melia F., Lund N., Paul J., Skinner G., Yusef-Zadeh F., 2006, ApJ, 636, 275 

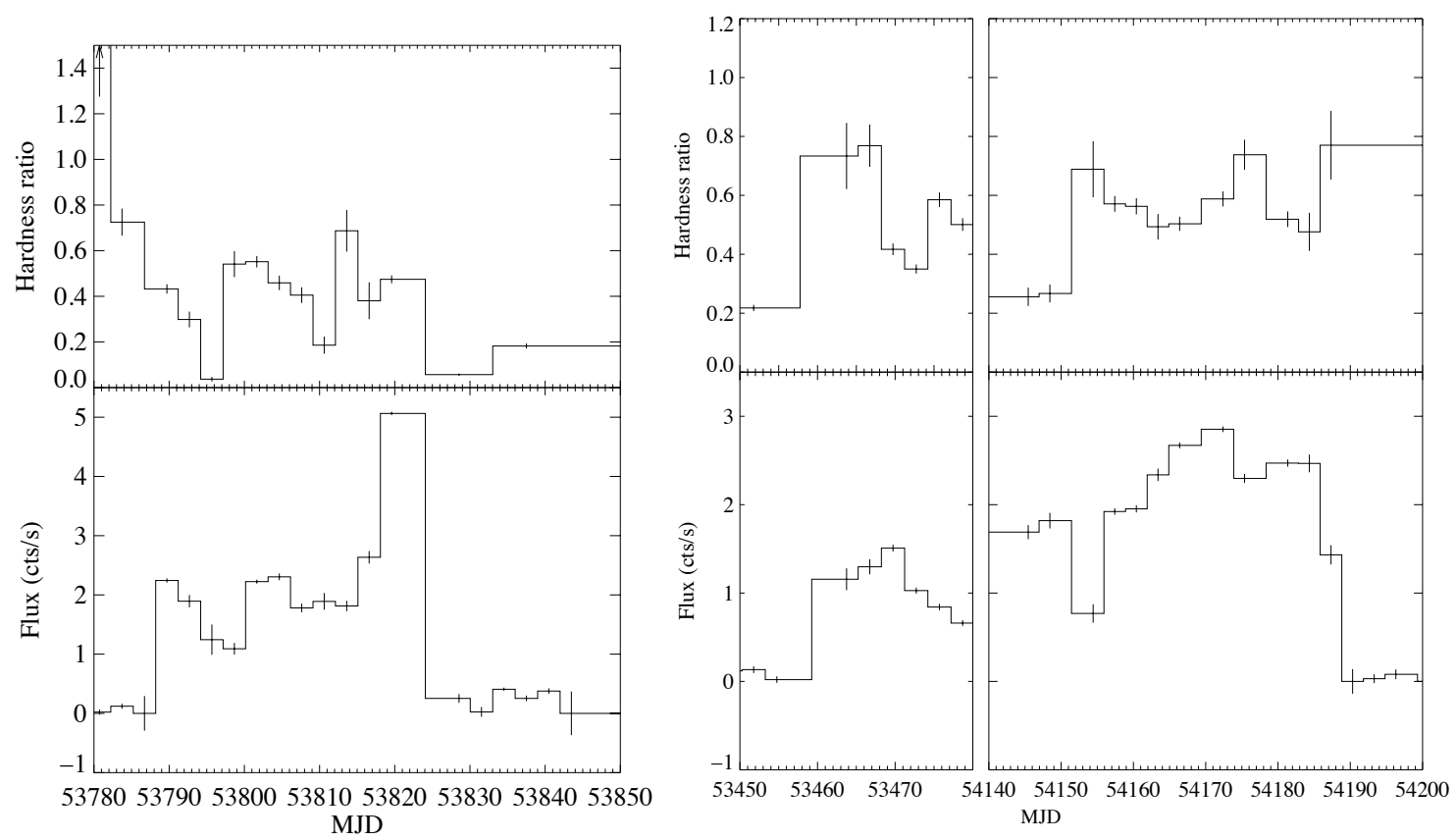

Figure 4: Hardness ratios (top) and $20-40 \mathrm{keV}$ flux lightcurves on revolution timescales for a single outburst of SAX J1747-2853 (left) and two outbursts of GRS 1741.9-2853 (right).

[2] Bélanger, G. and Goldwurm, A. and Goldoni, P. and Paul, J. and Terrier, R. and Falanga, M. and Ubertini, P. and Bazzano, A. and Del Santo, M. and Winkler, C. and Parmar, A. N. and Kuulkers, E. and Ebisawa, K. and Roques, J. P. and Lund, N. and Melia, F., 2004, ApJL, 601, 163

[3] Bird A. J., Bazzano A., Bassani L., Capitanio F., Fiocchi M., Hill A. B., Malizia A., McBride V. A., Scaringi S., Sguera V., Stephen J. B., Ubertini P., Dean, A. J.. Lebrun, F., Terrier, R., Renaud, M., Mattana, F., Götz, D., Rodriguez, J., Bélanger, G., Walter, R., Winkler, C., 2010, ApJS, 186, 1

[4] Kuulkers E., Shaw S. E., Paizis A., Chenevez J., Brandt S., Courvoisier T., Domingo A., Ebisawa K., Kretschmar P., Markwardt C. B., Mowlavi N., Oosterbroek T., Orr A., Rísquez D., Sanchez-Fernandez C., Wijnands R., 2007, A\&A, 466, 595

[5] Muno M. P., Baganoff F. K., Bautz M. W., Brandt W. N., Broos P. S., Feigelson E. D., Garmire G. P., Morris, M. R., Ricker, G. R., Townsley, L. K., 2003, ApJ, 589, 225

[6] Ubertini P., Lebrun F., Di Cocco G., Bazzano A., Bird A. J., Broenstad K., Goldwurm A., La Rosa G., Labanti C., Laurent P., Mirabel, I. F., Quadrini, E. M., Ramsey, B., Reglero, V., Sabau, L., Sacco, B., Staubert, R., Vigroux, L., Weisskopf, M. C., Zdziarski, A. A., 2003, A\&A, 411, L131 